\title{
Concurrent Validity and Reliability of a Handheld Dynamometer in Measuring Isometric Shoulder Rotational Strength
}

\author{
Bin Chen, Lifen Liu, Lincoln Bin Chen, Xianxin Cao, Peng Han, Chenhao Wang, and Qi Qi
}

\begin{abstract}
Context: Measuring isometric shoulder rotational strength is clinically important for evaluating motor disability in athletes with shoulder injuries. Recent evidence suggests that handheld dynamometry may provide a low-cost and portable method for the clinical assessment of isometric shoulder strength. Objective: To investigate the concurrent validity and the intrarater and interrater reliability of handheld dynamometry for measuring isometric shoulder rotational strength. Design: Cross-sectional study. Setting: Biomechanics laboratory. Participants: Thirty-nine young, healthy participants. Main Outcome Measures: The peak isometric strength of the internal rotators and external rotators, measured by handheld dynamometry (in newton) and isokinetic dynamometry (in newton meter). Interventions: Maximal isometric shoulder rotational strength was measured as participants lay supine with $90^{\circ}$ shoulder abduction, neutral rotation, $90^{\circ}$ elbow flexion, and forearm pronation. Measurements were performed independently by 2 different physiotherapists and in 3 different sessions to evaluate interrater and intrarater reliability. The data obtained by handheld dynamometry were compared with those obtained by isokinetic testing to evaluate concurrent validity. Results: The intraclass correlation coefficients for interrater reliability in measuring maximum isometric shoulder external and internal rotation strength were .914 (95\% confidence interval $[\mathrm{CI}], .842-.954)$ and $.842(95 \% \mathrm{CI}$, $.720-914)$, respectively. The intrarater reliability values of the method for measuring maximal shoulder external and internal rotation strength were 0.865 (95\% CI, 0.757-0.927) and 0.901 (95\% CI, 0.820-0.947), respectively. The Pearson correlation coefficients between the handheld and isokinetic dynamometer measurements were .792 (95\% CI, .575-.905) for external rotation strength and .664 (95\% CI, .419-.839) for internal rotation strength. Conclusions: The handheld dynamometer showed good to excellent reliability and moderate to good validity in measuring maximum isometric shoulder rotational strength. Therefore, handheld dynamometry could be acceptable for health and sports professionals in field situations to evaluate maximum isometric shoulder rotational strength.
\end{abstract}

Keywords: handheld dynamometry, isokinetic dynamometer, external, internal, portability

Isometric shoulder rotational strength is an important parameter that clinicians use to diagnose shoulder pathologies, monitor the progression and effectiveness of treatment, recognize risk factors, and implement prevention programs for shoulder activities. ${ }^{1}$ In addition, shoulder rotational strength measurements have been adopted to establish a normal profile, providing a valuable reference in overhead athletes and young swimmers. ${ }^{2}$ Therefore, it is particularly important for field professionals to have accurate and reliable assessment tools to measure shoulder rotational strength. ${ }^{3}$

Previous studies have shown that handheld dynamometry is superior to isokinetic testing, manual muscle testing, and externally fixed dynamometry in that it is cost effective and highly portable but still achieves strong concurrent validity and excellent reliability for isometric muscle strength assessment. ${ }^{3,4}$ A number of articles have examined isometric shoulder strength with different populations, muscle groups, testing positions, and devices, but conflicting results have been found for shoulder rotation. ${ }^{5,6}$ Therefore, the purpose of this study was to further evaluate the concurrent validity and the intrarater and interrater reliability of a new handheld dynamometer in measuring isometric shoulder rotational strength.

B. Chen, L.B. Chen, Cao, Han, Wang, and Qi are with the Department of Physical Therapy, Shanghai Yangzhi Rehabilitation Hospital, Tongji University School of Medicine, Shanghai, China. Liu is with the Tongji University School of Medicine, Shanghai, China. Qi (qibuqi133@163.com) is corresponding author.

\section{Methods}

This cross-sectional study evaluated the concurrent validity and reliability of a handheld dynamometer in measuring shoulder rotational strength.

Thirty-nine young, healthy participants (15 females and 24 males) participated in this study. All the participants in this investigation were recruited among the university students at Shanghai Sunshine Rehabilitation Center. The study design was approved by the institutional research ethics committee of Tongji University, all participants completed an informed consent form prior to assessment.

The inclusion criteria were as follows: (1) the participants had no shoulder injury or surgery and (2) the participants had no shoulder symptoms at the time of testing. The exclusion criterion was a history of neurological or musculoskeletal disease of the shoulder.

Maximum isometric shoulder rotational strength measurements were performed using a handheld dynamometer (MicroFET3; Hoggan Health Industries Inc, Biometrics, The Netherlands). During testing, the participants lay supine with the dominant shoulder abducted to $90^{\circ}$ and the elbow flexed to $90^{\circ}$, based on a previous article demonstrating practical application methods and body stabilization. The end piece of the dynamometer was placed against the distal forearm $2-\mathrm{cm}$ proximal to the styloid process with the forearm pronated and perpendicular to the floor. ${ }^{1}$ Manual stabilization of the upper arm was provided by the tester. To ensure the homogeneity 2 male physiotherapists (tester 1: height $=177 \mathrm{~cm}$, weight $=72 \mathrm{~kg}$; 
tester 2 : height $=180 \mathrm{~cm}$, weight $=65 \mathrm{~kg}$ ) conducted the strength measurement with 2-years' experience. The details of experimental design and data collection setup were shown in Figure 1.

In order to investigate the concurrent validity of the hand-held dynamometer, the isometric shoulder rotational strength was also tested with an isokinetic dynamometer (IsoMed-2000; D. \& R. Ferstl GmbH, Hemau, Germany) by tester 3 a week after the third session. All the measurements were performed as the participants lay supine with the shoulder abducted to $90^{\circ}$, the elbow flexed to $90^{\circ}$, and the forearm in neutral position. Three trials of 3 seconds of maximal isometric contraction were performed, and a 10 -second rest period between each measurement, and a 2-minute rest period was given between external and internal rotation measurements. The handheld dynamometer measured force in newtons, and the isokinetic dynamometer measured force in units of newton meters.

The peak isometric strength measurements across all sessions from the handheld dynamometer (in newton) and from the isokinetic dynamometer (in newton meter) were normalized to body weight for analysis. Means and SDs were calculated across participants for the dependent variables. All the variables were normally distributed (Kolmogorov-Smirnov test), and parametric tests were performed. For interrater reliability, the measurements of sessions 2 and 3 were used in consideration of familiarization from session 1 , and the intraclass correlation coefficient (ICC) value $\mathrm{ICC}_{2,1}$ (2-way random model and absolute agreement) was calculated. For intrarater reliability, the data from sessions 1 and 3 were used, and $\mathrm{ICC}_{3,1}$ (2-way mixed model, consistency) was calculated. Furthermore, the standard error of the mean with $95 \%$ confidence interval was used for interrater and intrarater reliability. The ICC values were classified as moderate (.50-.75), good (.76-.90), and excellent (>.90). ${ }^{7}$ Concurrent validity between measurements by the handheld dynamometer and the isokinetic dynamometer was determined by the correlation coefficient. Correlation values between .50 and .75 indicated a moderate to good relationship, and values above .75 indicated a good to excellent relationship. Values of $\geq .70$ were considered acceptable. ${ }^{8}$ The statistical analyses were run in IBM SPSS Statistics for Windows (version 22.0; IBM Corp, Armonk, NY).

\section{Results}

Table 1 shows the outcomes for maximal isometric shoulder rotational strength data from sessions 1,2 , and 3 . The handheld dynamometer protocol had good to excellent interrater reliability in measuring maximal shoulder internal rotation $(\mathrm{ICC}=.842$; $P<.001)$ and external rotation strength $(\mathrm{ICC}=.914 ; P<.001)$, respectively. The results of comparisons between sessions 1 and 3 ranged from 0.865 (external rotation) to 0.901 (internal rotation), showing good and excellent intrarater reliability, respectively.

Table 2 shows the maximal isometric shoulder rotational strength data measured by the handheld dynamometer and isokinetic dynamometer. The mean strength measured by the handheld dynamometer for external and internal rotation was 0.49 and $0.45 \mathrm{~N} \cdot \mathrm{m} / \mathrm{kg}$, respectively. The mean torque measured by the isokinetic dynamometer for external and internal rotation was 0.38 and $0.39 \mathrm{~N} \cdot \mathrm{m} / \mathrm{kg}$, respectively. Correlation coefficients ranged from .664 (internal rotation) to .792 (external rotation), indicating moderate to good correlation.
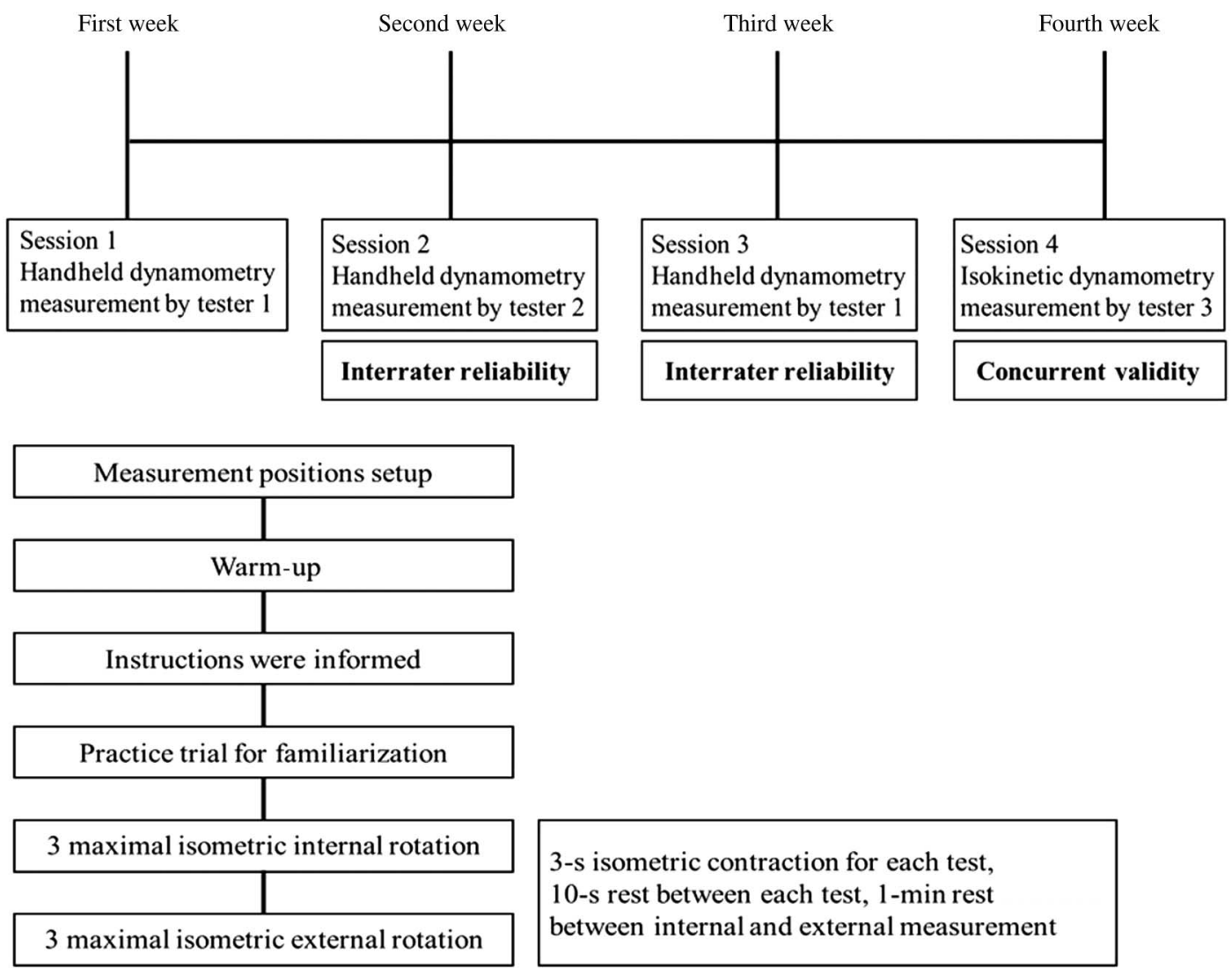

3-s isometric contraction for each test, 10 -s rest between each test, 1-min rest between internal and external measurement

Figure 1 - Experimental design of the study and data collection setup of handheld dynamometer. 
Table 1 Reliability Measures for Handheld Dynamometry

\begin{tabular}{|c|c|c|c|c|c|c|c|c|c|}
\hline \multirow{2}{*}{$\begin{array}{l}\text { Strength } \\
\text { values }(N / K G)^{b}\end{array}$} & \multirow[b]{2}{*}{ Session $1^{a}$} & \multirow[b]{2}{*}{ Session $2^{a}$} & \multirow[b]{2}{*}{ Session $3^{a}$} & \multicolumn{3}{|c|}{$\begin{array}{l}\text { Interrater reliability } \\
\text { (sessions } 2 \text { and } 3 \text { ) }\end{array}$} & \multicolumn{3}{|c|}{$\begin{array}{l}\text { Intrarater reliability } \\
\text { (sessions } 1 \text { and } 3 \text { ) }\end{array}$} \\
\hline & & & & ICC $(95 \% \mathrm{Cl})$ & SEM & $P$ value & ICC (95\% Cl) & SEM & $P$ value \\
\hline External rotation & $2.23(0.50)$ & $2.38(0.53)$ & $2.35(0.50)$ & $.914(.842-.954)$ & 0.15 & $P<.001$ & $.865(.757-.927)$ & 0.18 & $P<.001$ \\
\hline Internal rotation & $2.05(0.48)$ & $2.12(0.49)$ & $2.16(0.45)$ & $.842(.720-.914)$ & 0.19 & $P<.001$ & $.901(.820-.947)$ & 0.14 & $P<.001$ \\
\hline
\end{tabular}

Abbreviations: CI, confidence interval; ICC, intraclass correlation coefficient; SEM, standard error of the mean.

${ }^{\mathrm{a}}$ Values are given as the mean (SD). ${ }^{\mathrm{b}}$ Values normalized to body mass (in kilogram).

Table 2 Concurrent Validity Measures for Handheld Dynamometry

\begin{tabular}{|c|c|c|c|c|c|}
\hline Strength values & $\begin{array}{l}\text { Handheld dynamometry } \\
(\mathrm{N} \cdot \mathrm{m} / \mathrm{kg})^{\mathrm{a}, \mathrm{b}}\end{array}$ & $\begin{array}{l}\text { Isokinetic dynamometry } \\
(\mathrm{N} \cdot \mathrm{m} / \mathrm{kg})^{\mathrm{a}, \mathrm{b}}\end{array}$ & Pearson correlation & $95 \% \mathrm{Cl}$ & $P$ value \\
\hline External rotation & $0.49(0.12)$ & $0.38(0.10)$ & 0.792 & $0.575-0.905$ & $P<.001$ \\
\hline Internal rotation & $0.45(0.11)$ & $0.39(0.11)$ & 0.664 & $0.419-0.839$ & $P<.001$ \\
\hline
\end{tabular}

Abbreviation: CI, confidence interval.

${ }^{\mathrm{a}}$ Values are given as the mean (SD). ${ }^{\mathrm{b}}$ Values normalized to body mass (in kilogram).

\section{Discussion}

The main findings from this study showed that the handheld dynamometer provided a simple method to reliably measure shoulder rotational strength in clinical settings. The handheld dynamometer demonstrated good interrater reliability and good to excellent intrarater reliability in assessing isometric shoulder external and internal rotation strength. Furthermore, the isometric internal and external rotation strength measured by the handheld dynamometer showed moderate and good association, respectively, with isometric strength measured by the isokinetic dynamometer.

Despite the common use of externally fixed dynamometry, the stabilization devices used in handheld dynamometry and isokinetic dynamometry are not simple, portable, or accessible to many clinicians. ${ }^{3,4}$ For this reason, we proposed a reliable instrument that could be used more easily than other devices. To increase the efficiency of the application of handheld dynamometry in field situations, we performed all measurements of maximum isometric shoulder rotational strength without external fixation. It is worth noting that the intrarater (ICC .757-.947) and interrater reliability (ICC .720-.954) were good to excellent despite the use of minimal stabilization, which was similar to a previous study. ${ }^{9}$ Therefore, it is acceptable to measure shoulder rotational strength by handheld dynamometry without extra stabilization between trials, meaning that the protocol could be easily adopted in clinical settings.

A previous study reported excellent concurrent reliability (internal rotation: $r=.991$; external rotation: $r=.998$ ) when measuring isometric shoulder rotational strength using a standard handheld dynamometer; these values are higher than what was found in the present study (internal rotation: $r=.664$; external rotation: $r=.792){ }^{4}$ In contrast to our methods, however, isometric strength was measured via an externally fixed dynamometer in that study, or the shoulder was stabilized by a belt. The advantage of handheld dynamometry described in this paper is that the device is portable and efficient for measurement in clinical settings; therefore, we did not physically restrict the shoulder for handheld dynamometry, which makes the technique more sophisticated and challenging for some clinicians.

Regarding the concurrent validity of rotational strength measurement, the correlation values and 95\% confidence interval reported for shoulder internal rotation were generally lower and wider, respectively, than those for external rotation. A possible reason for previous observations was that the participants substituted more easily when performing internal rotation. In addition, the latissimus dorsi and pectoralis major act with the teres major to provide the main force of shoulder internal rotation. ${ }^{10}$ Therefore, substitution during the internal rotation measurement may be due to the adduction moment of the latissimus dorsi and pectoralis major.

This study has several limitations. First, we chose positions that were conducive to portable and efficient testing in order to simplify the process compared with handheld dynamometry measurements with external fixation; the lack of external fixation would lower our observed correlations. Future studies could therefore evaluate the fixed 90-90 position for handheld dynamometry and isokinetic profiles of shoulder rotational strength. Second, all the participants in this study were healthy university students. Thus, the results may not be highly generalizable to athletes with shoulder pathology. Finally, given the large groups of rotational muscles around the shoulder, some participants may have compensated using the trunk during the testing, although the participants received instructions and performed practice trials before the measurements.

\section{Conclusions}

Measurements of maximum isometric shoulder rotational strength by handheld dynamometry in the present study showed good to excellent reliability and moderate to good concurrent validity in healthy participants. Therefore, handheld dynamometry could be suitable for health and sports professionals in field situations to evaluate shoulder rotational strength due to the portability and ease of use of the equipment.

\section{Acknowledgment}

As there has been no financial support for this project, the authors are grateful to the volunteers who participated in this study.

\section{References}

1. Byram IR, Bushnell BD, Dugger K, Charron K, Harrell FE Jr, Noonan TJ. Preseason shoulder strength measurements in professional baseball 
pitchers: identifying players at risk for injury. Am J Sports Med. 2010;38(7):1375-1382. PubMed ID: 20489215 doi:10.1177/036354 6509360404

2. McLaine SJ, Ginn KA, Fell JW, Bird ML. Isometric shoulder strength in young swimmers. J Sci Med Sport. 2018;21(1):35-39. PubMed ID: 28844605 doi:10.1016/j.jsams.2017.05.003

3. Cadogan A, Laslett M, Hing W, McNair P, Williams M. Reliability of a new hand-held dynamometer in measuring shoulder range of motion and strength. Man Ther. 2011; 16(1):97-101. PubMed ID: 20621547 doi:10.1016/j.math.2010.05.005

4. Romero-Franco N, Fernandez-Dominguez JC, Montano-Munuera JA, Romero-Franco J, Jimenez-Reyes P. Validity and reliability of a low-cost dynamometer to assess maximal isometric strength of upper limb. J Sports Sci. 2019;37(15):1787-1793. PubMed ID: 30897030 doi:10.1080/02640414.2019.1594570

5. Beshay N, Lam PH, Murrell AC. Assessing the reliability of shoulder strength measurement hand-held versus fixed dynamometry. Shoulder Elbow. 2011;3(4):244-251. doi:10.1111/j.1758-5740. 2011.00137.x
6. Riemann BL, Davies GJ, Ludwig L, Gardenhour H. Hand-held dynamometer testing of the internal and external rotator musculature based on selected positions to establish normative data and unilateral ratios. J Shoulder Elbow Surg. 2010;19(8):1175-1183. PubMed ID: 20850997 doi:10.1016/j.jse.2010.05.021

7. de Vet HC, Terwee CB, Knol DL, Bouter LM. When to use agreement versus reliability measures. J Clin Epidemiol. 2006; 59(10):1033-1039. PubMed ID: 16980142 doi:10.1016/j.jclinepi. 2005.10.015

8. Portney LG, Watkins MP. Foundations of Clinical Research: Applications to Practice. 3rd ed. Upper Saddle River, NJ: Pearson/ Prentice Hall: F. A. Davis PT; 2015.

9. McLaine SJ, Ginn KA, Kitic CM, Fell JW, Bird ML. The reliability of strength tests performed in elevated shoulder positions using a handheld dynamometer. J Sport Rehabil. 2016;25(2):2015. PubMed ID: 26355715 doi:10.1123/jsr.2015-0034

10. Hess SA. Functional stability of the glenohumeral joint. Man Ther. 2000;5(2):63-71. PubMed ID: 10903581 doi:10.1054/math.2000. 0241 